PROCEEDINGS OF THE

AMERICAN MATHEMATICAL SOCIETY

Volume 140, Number 9, September 2012, Pages 3055-3066

S 0002-9939(2011)11131-6

Article electronically published on December 28, 2011

\title{
ON THE WAVE OPERATOR FOR THE GENERALIZED BOUSSINESQ EQUATION
}

\author{
LUIZ GUSTAVO FARAH AND LUCAS C. F. FERREIRA
}

(Communicated by Walter Craig)

\begin{abstract}
We study the asymptotic behavior of solutions for the generalized Boussinesq equation in a singular framework. We construct a wave operator (inverse scattering) for large profiles $\vec{h}$ belonging to an infinite mass framework based on weak $L^{p}$-spaces. Our solutions converge towards the prescribed scattering state with a polynomial rate.
\end{abstract}

\section{INTRODUCTION}

In this work we concern ourselves with the generalized Boussinesq equation

$$
\begin{aligned}
& u_{t t}-\Delta u+\Delta^{2} u+\Delta f(u)=0, \quad x \in \mathbb{R}^{n}, t>0, \\
& u(x, 0)=\phi, \quad u_{t}(x, 0)=\psi,
\end{aligned}
$$

where $f: \mathbb{R} \rightarrow \mathbb{R}, f(0)=0$ and there is $C_{f}>0$ such that

$$
|f(a)-f(b)| \leq C_{f}|a-b|\left(|a|^{\rho-1}+|b|^{\rho-1}\right), \quad \forall a, b \in \mathbb{R},
$$

for a given $1<\rho<\infty$. A typical example of (1.2) is $f(u)=u|u|^{\rho-1}$. The equation (1.1) is equivalent to the system of equations

$$
\left\{\begin{array}{l}
u_{t}=\Delta v \\
v_{t}=u-\Delta u-f(u), \quad x \in \mathbb{R}^{n}, t>0 .
\end{array}\right.
$$

We can also write the integral equation for the above system. First, recall that the solution of the linear system associated to (1.3) with initial data $\vec{u}_{0}=\left(u_{0,1}, u_{0,2}\right)$ is given by

$$
B(t) \vec{u}_{0}=\int_{-\infty}^{+\infty} e^{i x \xi}\left(\begin{array}{cc}
\cos (t|\xi|\langle\xi\rangle) & -\frac{|\xi|}{|\xi \xi\rangle} \sin (t|\xi|\langle\xi\rangle) \\
\frac{\langle\xi\rangle}{|\xi|} \sin (t|\xi|\langle\xi\rangle) & \cos (t|\xi|\langle\xi\rangle)
\end{array}\right) \widehat{\vec{u}_{0}}(\xi) d \xi,
$$

where $\langle\xi\rangle=\left(1+|\xi|^{2}\right)^{1 / 2}$ and $\widehat{\vec{u}_{0}}=\left(\widehat{u}_{0,1}, \widehat{u}_{0,2}\right)$. In view of Duhamel principle, solutions of (1.1) and (1.3) can be rewritten as

$$
\vec{u}(t)=B(t) \vec{u}_{0}+\int_{0}^{t} B\left(t-t^{\prime}\right) \vec{f}\left(\vec{u}\left(t^{\prime}\right)\right) d t^{\prime},
$$

where $\vec{f}(\vec{u})=(0, f(u))$ and $\vec{u}=\left(u_{1}, u_{2}\right)=(u, v)$.

Received by the editors October 1, 2010 and, in revised form, March 16, 2011.

2010 Mathematics Subject Classification. Primary 35Q35; Secondary 35B40, 35P25.

Key words and phrases. Boussinesq equation, inverse scattering, large data, Lorentz spaces.

The first author was partially supported by FAPEMIG and CNPq, Brazil.

The second author was supported by CNPq and FAPESP, Brazil.

(C)2011 American Mathematical Society 
This kind of equation occurs in a large number of physical situations, which has motivated their study through physical and mathematical approaches. Initially, in one space dimension and with opposite sign in the term $\Delta^{2} u$, equation (1.1) was employed by Boussinesq [3] to model nonlinear shallow water waves. Later on, further applications appeared by describing dynamics of stretched string, shapememory alloys, ion-sound waves, and many others (see e.g. [7, 4, 16]).

The issues of existence and asymptotic behavior of solutions for the generalized Boussinesq equation (1.1) have been addressed by several authors. In the framework of Sobolev spaces, we refer the reader to [2, 13, 18, 9, 10] for local or global wellposedness results and [14] for small scattering theory. In [4, 15], the authors proved existence results by employing certain Besov spaces with positive regularity. In these two works, a scattering theory for small solutions was also developed together with some decay rates for the norms involved. The reader is also referred to [5, 17, 19] for existence and scattering results in Sobolev spaces involving other dispersive systems connected to (1.1). Next we briefly explain the scattering problem. Given a nonlinear PDE, the problem consists of finding suitable $V$ such that the solution $u_{V}(t)$ of the linear problem associated to this PDE with initial data $V$ describes the asymptotic behavior of its global solution with small data $\phi$ and $\psi$; precisely, $\lim _{t \rightarrow+\infty}\left\|u(t)-u_{V}(t)\right\|_{X}=0$, where $X$ is a Banach space.

Another interesting question studied for dispersive models is the reciprocal problem of the scattering theory, namely inverse scattering, which consists of constructing a solution with a prescribed scattering state. More roughly, for a given profile $V$ (regardless of its size), one looks for a solution of the nonlinear problem $u(t)$, defined for large enough $t$, such that

$$
\lim _{t \rightarrow \infty}\left\|u(t)-u_{V}(t)\right\|_{Y}=0
$$

where $u_{V}(t)$ is the solution of the linear problem with initial data $V$ and $Y$ stands for a suitable Banach space. Solving the latter problem is also known as the construction of a wave operator. Inverse scattering was studied in Sobolev or weighted Sobolev spaces for Schrödinger equations (see e.g. [11, 12]) and for generalized Korteweg-de Vries equations in [6]. By using the integral formulation (1.5), the first author 8 studied inverse scattering through a $H_{p}^{s}$-framework $(s>0)$.

In the above mentioned papers, although the smallness assumption on the profile $V$ has been removed, finite $L^{p}$-norm and some positive regularity on $V$ are necessary to obtain some scattering or inverse scattering results. The reason comes from the needed estimates to perform a contraction argument in the corresponding frameworks.

In this paper, our aim is to construct a large data wave operator for the generalized Boussinesq equation (1.1) by means of the integral formulation (1.5) on weak $L^{p}$-type spaces. In this kind of space the elements, in general, have infinite $L^{p}$-norm, for all $1 \leq p \leq \infty$, and particularly infinite mass, that is $\|\cdot\|_{L^{2}}=\infty$. Therefore, our framework does not make use of positive regularity, and it contains interesting singular functions, including some with infinite local mass (see Remark 2.3 below). Our main result, given in Theorem [2.2, extends the results in 8 in the sense that the admissible profile class $\mathcal{I}_{\sigma}^{T}$ is strictly larger; see Remark 2.2 below for details. Our solutions go towards the prescribed scattering states with polynomial-time rates. 
The approach used here also generalizes those employed in [8, 6] in the sense that it is easy to see that the family of linear operators $\{B(t)\}_{t \in \mathbb{R}}$ given in (1.4) verifies (at least formally) the group property with operation being the composition. Moreover, in view of Lemma 3.1 below, we are dealing with a framework in which the linear group $B(t)$ is not unitary; that is, it does not preserve weak $L^{p}$-norms. In the previously mentioned papers the authors dealt with certain Besov and Sobolev norms in which $B(t)$ is a unitary group.

The plan of this paper is the following: In Section 2, we introduce some notation, review facts about Lorentz spaces, and state our results. We provide some linear estimates in Section 3. Finally, the proofs of the results are given in Section 4.

\section{Notation AND MAin RESUlts}

We start by recalling some definitions and properties on Lorentz spaces. For more details we refer the reader to [1. The Lorentz space $L^{(p, q)}$ is a Banach space of measurable functions endowed with the norm $\|\cdot\|_{(p, q)}$ given by

$$
\|f\|_{(p, q)}= \begin{cases}\left(\frac{p}{q} \int_{0}^{\infty}\left[t^{\frac{1}{p}} f^{* *}(t)\right]^{q} \frac{d t}{t}\right)^{\frac{1}{q}}, & \text { if } \quad 1<p<\infty, 1 \leq q<\infty, \\ \sup _{t>0} t^{\frac{1}{p}} f^{* *}(t), & \text { if } \quad 1<p \leq \infty, q=\infty,\end{cases}
$$

and

$$
f^{* *}(t)=\frac{1}{t} \int_{0}^{t} f^{*}(s) d s \text { and } f^{*}(t)=\inf \left\{s>0: m\left\{x \in \mathbb{R}^{n}:|f(x)|>s\right\} \leq t\right\},
$$

where $m$ stands for the Lebesgue measure in $\mathbb{R}^{n}$. The weak $L^{p}$-spaces correspond to the special case $q=\infty$ (i.e. $L^{(p, \infty)}$ ) and they are also known as Marcinkiewicz spaces. In view of the continuous inclusions

$$
L^{(p, 1)} \subset L^{\left(p, q_{1}\right)} \subset L^{(p, p)}=L^{p} \subset L^{\left(p, q_{2}\right)} \subset L^{(p, \infty)},
$$

for all $1 \leq q_{1} \leq p \leq q_{2} \leq \infty, L^{(p, \infty)}$ is the largest space in the family $\left\{L^{(p, q)}\right\}_{1 \leq q \leq \infty}$. The Holder inequalities also hold in this framework, namely

$$
\|h\|_{(r, s)} \leq C(r)\|f\|_{\left(p_{1}, q_{1}\right)}\|g\|_{\left(p_{2}, q_{2}\right)},
$$

where $1<p_{1}, p_{2}<\infty, \frac{1}{r}=\frac{1}{p_{1}}+\frac{1}{p_{2}}, s \geq 1$ and $\frac{1}{q_{1}}+\frac{1}{q_{2}} \geq \frac{1}{s}$. From the interpolation point of view, we have $L^{(p, q)}=\left(L^{1}, L^{\infty}\right)_{1-\frac{1}{p}, q}$ with $1<p<\infty$ and $1 \leq q \leq \infty$. Moreover, it follows from the Reiteration Theorem that

$$
\left(L^{\left(p_{0}, q_{0}\right)}, L^{\left(p_{1}, q_{1}\right)}\right)_{\theta, q}=L^{(p, q)},
$$

provided that $1<p_{0}<p_{1}<\infty, 0<\theta<1, \frac{1}{p}=\frac{1-\theta}{p_{0}}+\frac{\theta}{p_{1}}$ and $1 \leq q_{0}, q_{1}, q \leq \infty$.

To state our results we also need to define the following product spaces based on the Lorentz spaces.

Definition 2.1. Let $D^{s}=(-\Delta)^{s / 2}$ and $J^{s}=(1-\Delta)^{s / 2}$. Consider the Banach space

$$
X_{(p, d)}=L^{(p, d)} \times D^{-1} J\left(L^{(p, d)}\right)
$$

endowed with the norm

$$
\left\|\left[g_{1}, g_{2}\right]\right\|_{X_{(p, d)}}=\max \left\{\left\|g_{1}\right\|_{(p, d)},\left\|D J^{-1} g_{2}\right\|_{(p, d)}\right\} .
$$


Given $0 \leq \sigma<\infty$ and $0<T<\infty$, define $\mathcal{E}_{\sigma}^{T}$ to be the space of Bochner measurable vectors $\vec{u}(t)=\left(u_{1}(t), u_{2}(t)\right) \vec{u}:(-\infty, \infty) \rightarrow X_{(\rho+1, \infty)}$ with norm

$$
\|\vec{u}\|_{\mathcal{E}_{\sigma}^{T}} \equiv \sup _{t>T} t^{\sigma}\|\vec{u}(\cdot, t)\|_{X_{(\rho+1, \infty)}} .
$$

We also define the profile space $\mathcal{I}_{\sigma}^{T}$ as the set of vectors $\vec{h}=\left(h_{1}, h_{2}\right) \in \mathcal{S}^{\prime}\left(\mathbb{R}^{n}\right) \times$ $\mathcal{S}^{\prime}\left(\mathbb{R}^{n}\right)$ satisfying

$$
\|\vec{h}\|_{\mathcal{I}_{\sigma}^{T}}=\sup _{t>T} t^{\sigma}\|B(t) \vec{h}\|_{X_{(\rho+1, \infty)}}<\infty .
$$

Henceforth $\gamma(n)=\infty$ if $n=1,2$ and $\gamma(n)=\frac{n+2}{n-2}$, otherwise. This parameter seems to have a universal role and appears frequently in the literature related to the Sobolev critical exponent.

Furthermore, we denote $\beta=n(\rho-1) / 2(\rho+1), \alpha=1 /(\rho-1)-n / 2(\rho+1)$, and $\rho_{0}(n)=\frac{n+2+\sqrt{n^{2}+12 n+4}}{2 n}$ the positive root of $n \rho^{2}-(n+2) \rho-2=0$. Notice that $\rho_{0}(n)<\rho$ is equivalent to $\alpha<\beta$.

Now we can give a precise statement of our results.

Theorem 2.1. Let $\rho_{0}(n)<\rho<\gamma(n)$ and $\alpha<\sigma<\beta$. For any $\vec{h}=\left(h_{1}, h_{2}\right) \in$ $L^{\left(\frac{\rho+1}{\rho}, \infty\right)} \times L^{\left(\frac{\rho+1}{\rho}, \infty\right)}$, there exist $T_{0}=T_{0}(\vec{h})>0$ and a solution $\vec{u} \in \mathcal{E}_{\sigma}^{T_{0}}$ of the integral equation (1.5) such that

$$
\lim _{t \rightarrow \infty} t^{\sigma}\|\vec{u}(t)-B(t) \vec{h}\|_{X_{(\rho+1, \infty)}}=0 .
$$

Moreover, $\vec{u}$ is the unique solution such that

$$
\sup _{t>T} t^{\sigma}\|\vec{u}(t)\|_{X_{(\rho+1, \infty)}}<\infty, \text { for some } T \geq T_{0} .
$$

Actually, a slight modification on the above theorem allows us to relax the hypotheses on $\rho, \sigma$ and $\vec{h}$ and to improve the decay given in (2.7). This is the content of the following theorem.

Theorem 2.2. Let $1<\rho<\gamma(n), \alpha<\sigma<\infty$, and $\widetilde{T}_{0}>0$. For any $\vec{h}=\left(h_{1}, h_{2}\right) \in$ $\mathcal{I}_{\sigma}^{\widetilde{T}_{0}}$ there exist $T_{0}=T_{0}(\vec{h}) \geq \widetilde{T}_{0}$ and a solution $\vec{u} \in \mathcal{E}_{\sigma}^{T_{0}}$ of the integral equation (1.5) such that

$$
\lim _{t \rightarrow \infty} t^{\sigma}\|\vec{u}(t)-B(t) \vec{h}\|_{X_{(\rho+1, \infty)}}=0 .
$$

Moreover, assume that $\vec{v} \in \mathcal{E}_{\sigma}^{T_{0}}$ is another solution with profile $\vec{h} \in \mathcal{I}_{\sigma}^{\widetilde{T}_{0}}$. Then $\vec{u}=\vec{v}$ in $[T, \infty)$, for some $T \geq T_{0}$.

Below we compare the decay rates obtained in the above theorems with the decay rate of the free Boussinesq equation.

Remark 2.1. Recall that the solution of the free Boussinesq equation with data $\left[u_{0}, v_{0}\right]$ is given by $B(t)\left[u_{0}, v_{0}\right]$. For an arbitrary $\vec{h} \in L^{\left(\frac{\rho+1}{\rho}, \infty\right)} \times L^{\left(\frac{\rho+1}{\rho}, \infty\right)}$, it follows from Lemma 3.1 below that $\left\|B(t)\left[u_{0}, v_{0}\right]\right\|_{X_{(\rho+1, \infty)}}=o\left(t^{-\sigma}\right)$ holds true only in the case $\sigma<\beta$. Indeed, the latter decay is not verified when $\sigma=\beta$ and $\vec{h}$ is as in Remark 2.3. Therefore, in view of Theorem 2.1, the solution $\vec{u}(t)$ goes to $B(t) \vec{h}$ with the same decay rate of solutions of the free Boussinesq equation. On the other hand, in Theorem 2.2 the constraint $\sigma<\beta$ has been removed by taking the profile $\vec{h} \in \mathcal{I}_{\sigma}^{\widetilde{T}_{0}}$. However, from an easy-handling point of view, the characterization of elements of $L^{\left(\frac{\rho+1}{\rho}, \infty\right)} \times L^{\left(\frac{\rho+1}{\rho}, \infty\right)}$ is better than that of $\mathcal{I}_{\sigma}^{\widetilde{T}_{0}}$. 
In the sequel we compare our profile class with that of $[\underline{8}$.

Remark 2.2. Recall the notation $\dot{B}_{p, q}^{s}$ for homogeneous Besov spaces and define the operator $\left(D_{1}(\psi)\right)^{\wedge}=\left(|\xi|\langle\xi\rangle^{-1}\right)^{\frac{(n-2)}{2}} \widehat{\psi}$. The author of [8] considered profiles $\vec{h} \epsilon$ $Y_{2}^{1} \cap Y_{\frac{\rho+1}{\rho}}^{1}$ with $\rho>2+\sqrt{7}$ for $n=1$, and $\vec{h} \in \mathfrak{B}_{\frac{\rho+1}{\rho}, 2}^{\frac{\rho-1}{\rho+1}, s}$ with $\rho_{0}(n)<\rho<\gamma(n)$ for $n>1$, where $Y_{p}^{s}=H_{p}^{s} \times D^{-1} J\left(H_{p}^{s}\right), \mathfrak{B}_{p, q}^{s}=N_{p, q}^{s} \times D^{-1} J\left(N_{p, q}^{s}\right)$ and $N_{p, q}^{s}=$ $\left(L^{p}\right) \cap D_{1}^{-l}\left(\dot{B}_{p, q}^{s}\right)$. Taking $\alpha<\sigma \leq \beta$, our profile class contains that of [8], since

$$
Y_{\frac{\rho+1}{\rho}}^{1}, \mathfrak{B}_{\frac{\rho+1}{\rho}, 2}^{\frac{\rho-1}{\rho+1}, s} \subset L^{\frac{\rho+1}{\rho}} \times D^{-1} J\left(L^{\frac{\rho+1}{\rho}}\right) \subset \mathcal{I}_{\sigma}^{T} .
$$

Indeed, using the inclusions $L^{\rho+1} \subset L^{(\rho+1, \infty)}$ and $L^{\rho+1} \times D^{-1} J\left(L^{\rho+1}\right) \subset X_{(\rho+1, \infty)}$, and afterwards (3.7), we obtain

$$
\begin{aligned}
& \|\vec{h}\|_{\mathcal{I}_{\sigma}^{T}}=\sup _{t>T} t^{\sigma}\|B(t) \vec{h}\|_{X_{(\rho+1, \infty)}} \leq C \sup _{t>T} t^{\sigma}\|B(t) \vec{h}\|_{L^{\rho+1} \times D^{-1} J\left(L^{\rho+1}\right)} \\
& \leq C\left(\sup _{t>T} t^{\sigma-\beta}\right) \max \left\{\left\|h_{1}\right\|_{L^{\frac{\rho+1}{\rho}}},\left\|h_{2}\right\|_{L^{\frac{\rho+1}{\rho}}}\right) \leq C\|\vec{h}\|_{\substack{\frac{\rho-1}{\rho+1}, s \\
\frac{\rho+1}{\rho}, 2}},
\end{aligned}
$$

which yields (2.9). A similar analysis holds for the space $Y_{\frac{\rho+1}{\rho}}^{1}$.

In the next remark we provide examples of infinite mass profile $\vec{h}$ covered by our class defined in (2.6)

Remark 2.3 (Singular profiles with infinite mass). In view of (3.1), if $\alpha<\sigma \leq \beta$, then the continuous inclusion $L^{\left(\frac{\rho+1}{\rho}, \infty\right)} \times L^{\left(\frac{\rho+1}{\rho}, \infty\right)} \subset \mathcal{I}_{\sigma}^{T_{0}}$ holds true for any $T_{0}>0$. Hence (in particular) the space $L^{\left(\frac{\rho+1}{\rho}, \infty\right)} \times L^{\left(\frac{\rho+1}{\rho}, \infty\right)}$ is an admissible profile class for $\vec{h}$ in Theorem 2.2 and contains singular profiles $\vec{h} \notin L_{\text {loc }}^{2}$ and $\vec{h} \notin L^{p}$ for all $1 \leq p \leq \infty$. To see this, let $\nu_{i}, \eta_{j}$ be constants satisfying $\sum_{i=1}^{\infty}\left|\nu_{i}\right|+\sum_{j=1}^{\infty}\left|\eta_{j}\right|<\infty$, and consider $\vec{h}=\left(h_{1}, h_{2}\right)$ such that

$h_{1}=\sum_{i=1}^{\infty} \nu_{i} P_{k, i}\left(x-x_{i}\right)\left|x-x_{i}\right|^{-k-\frac{n \rho}{\rho+1}}$ and $h_{2}=\sum_{j=1}^{\infty} \eta_{j} Q_{s, j}\left(x-\tilde{x}_{j}\right)\left|x-\tilde{x}_{j}\right|^{-s-\frac{n \rho}{\rho+1}}$,

where $x_{i}, \tilde{x}_{j} \in \mathbb{R}^{n}$ and $P_{k, i}(x), Q_{s, j}(x)$ are homogeneous polynomials with degrees $k, s$, respectively.

Remark 2.4 (Extension to $t=0$ ). Due to the high singularity of the spaces that we deal with, it is expected that blow-up will occur when extending the solution from $T$ to $t=0$. The usual way to perform such an extension is to handle suitable conservative quantities which are available only on Sobolev or Lebesgue spaces. In fact, there are no known conservative quantities involving weak $L^{p}$-norms, and it seems to be improbable to obtain those quantities, or, at least, it would be a very difficult task.

In the next theorem, we obtain a stronger convergence than those given in (2.7) and (2.8) by taking $\vec{h}$ in a more restricted space. Note that $X_{(\rho+1, d)} \subset X_{(\rho+1, \infty)}$ for all $1 \leq d \leq \infty$ in view of (2.2). 
Theorem 2.3. Assume that $1 \leq d \leq \infty$ and either the hypotheses of Theorem 2.1 or Theorem 2.2 hold. There exists $T \geq T_{0}$ such that if

$$
\sup _{t>T} t^{\sigma}\|B(t) \vec{h}\|_{X_{(\rho+1, d)}}<\infty
$$

then the previous solution $\vec{u} \in \mathcal{E}_{\sigma}^{T_{0}}$ of the integral equation (1.5) satisfies

$$
\lim _{t \rightarrow \infty} t^{\sigma}\|\vec{u}(t)-B(t) \vec{h}\|_{X_{(\rho+1, d)}}=0 .
$$

In particular, condition (2.11) holds true when $\vec{h} \in L^{\left(\frac{\rho+1}{\rho}, d\right)} \times L^{\left(\frac{\rho+1}{\rho}, d\right)}$ and $\alpha \leq \sigma \leq$ $\beta$.

Assuming further regularity on $\vec{h}$, we can also obtain convergence of the derivatives of $\vec{u}$ to those of the scattering state $B(t) \vec{h}$. Precisely, we have the following remark.

Remark 2.5. Let $[\rho]$ be the integer part of $\rho, f \in C^{[\rho]}(\mathbb{R}), m \in \mathbb{N}$, and let $k \in$ $(\mathbb{N})^{n}$ be a multi-index. Let $\nabla_{x}^{k}$ denote derivatives of order $|k|$. Assume also that $\left|f^{(l)}(v)\right| \lesssim|v|^{\rho-l}$ for all integers $l$ such that $0 \leq l \leq \rho$. Let $\mathcal{E}_{\sigma, m}^{T}$ be the Banach space of Bochner measurable vectors with norm

$$
\|\vec{u}\|_{\mathcal{E}_{\sigma, m}^{T}} \equiv \max _{|k| \leq m} \sup _{t>T} t^{\sigma}\left\|\nabla_{x}^{k} \vec{u}(x, t)\right\|_{X_{(\rho+1, \infty)}},
$$

and $\mathcal{I}_{\sigma, m}^{T}$ be the set of $\vec{h} \in \mathcal{S}^{\prime}\left(\mathbb{R}^{n}\right) \times \mathcal{S}^{\prime}\left(\mathbb{R}^{n}\right)$ satisfying

$$
\|\vec{h}\|_{\mathcal{I}_{\sigma, m}^{T}}=\max _{|k| \leq m} \sup _{t>T} t^{\sigma}\left\|\nabla_{x}^{k} B(t) \vec{h}\right\|_{X_{(\rho+1, \infty)}}<\infty
$$

Assuming $\vec{h} \in \mathcal{I}_{\sigma, m}^{\widetilde{T}_{0}}$ instead of $\quad \mathcal{I}_{\sigma, 0}^{\widetilde{T}_{0}}=\mathcal{I}_{\sigma}^{\widetilde{T}_{0}}$ and adapting the proofs of Theorems 2.1 and 2.2 one can prove that the previous solution $\vec{u}$ belongs to $\mathcal{E}_{\sigma, m}^{T_{0}}$ for some $T \geq \widetilde{T}_{0}$, and

$$
\lim _{t \rightarrow \infty} t^{\sigma}\left\|\nabla_{x}^{k} \vec{u}(t)-\nabla_{x}^{k} B(t) \vec{h}\right\|_{X_{(\rho+1, \infty)}}=0, \text { for all }|k| \leq m,
$$

provided that either $\rho$ is an integer or $|k| \leq \rho-1$.

\section{LinEAR ESTIMATES}

In the next lemma we state some linear estimates for the group $B(\cdot)$ defined in (1.4) on Lorentz spaces. From now on, the notation $C>0$ will denote generic positive constants which may change line by line or even within the same line. We denote by $\|\vec{h}\|_{(p, d)}=\max \left\{\left\|h_{1}\right\|_{(p, d)},\left\|h_{2}\right\|_{(p, d)}\right\}$ the norm in $L^{(p, q)} \times L^{(p, q)}$. In the next lemma we state some linear estimates for the group $B(\cdot)$ defined in (1.4) on Lorentz spaces.

Lemma 3.1. Let $1 \leq d \leq \infty, 1<p \leq 2$, and $p^{\prime}$ such that $\frac{1}{p}+\frac{1}{p^{\prime}}=1$. Then there exists a positive constant $C=C(n, p)$ such that

$$
\|B(t) \vec{h}\|_{X_{\left(p^{\prime}, d\right)}} \leq C|t|^{-\frac{n}{2}\left(\frac{2}{p}-1\right)}\|\vec{h}\|_{(p, d)},
$$

for all $\vec{h} \in L^{(p, q)} \times L^{(p, q)}$ and $t \neq 0$. 
Proof. Let us first define the multiplier operators $l_{1}(t)$ and $l_{2}(t)$ with symbols $\cos (t|\xi|\langle\xi\rangle)$ and $\sin (t|\xi|\langle\xi\rangle)$, respectively. Recall the $L^{r}-L^{r^{\prime}}$ estimate (see e.g. [4])

$$
\left\|l_{i}(t) \varphi\right\|_{L^{r^{\prime}}} \leq C|t|^{-\frac{n}{2}\left(\frac{2}{r}-1\right)}\|\varphi\|_{L^{r}}, i=1,2
$$

and the notation $\vec{h}=\left(h_{1}, h_{2}\right)$. We express $B(t) \vec{h}=\left(\pi_{1} B(t) \vec{h}, \pi_{2} B(t) \vec{h}\right)$, where $\pi_{i}$ is the $i$-th projection, $i=1,2$. It follows from definition (1.4) and inequality (3.2) that

$$
\left\|\pi_{1} B(t) \vec{h}\right\|_{L^{p^{\prime}}} \leq C|t|^{-\frac{n}{2}\left(\frac{2}{p}-1\right)} \max \left\{\left\|h_{1}\right\|_{L^{p}},\left\|h_{2}\right\|_{L^{p}}\right\} \text { for all } t \neq 0,
$$

where $C>0$ depends only on $n, p$. Again by definition (1.4), we have

$$
D J^{-1} \pi_{2} B(t) \vec{h}=l_{2}(t) h_{1}+D J^{-1} l_{1}(t) h_{2} .
$$

Moreover, by $L^{r}$-continuity of the operator $D J^{-1}$ and (3.2), it follows that

$$
\begin{aligned}
\left\|\pi_{2} B(t) \vec{h}\right\|_{D^{-1} J\left(L^{p^{\prime}}\right)} & =\left\|D J^{-1} \pi_{2} B(t) \vec{h}\right\|_{L^{p^{\prime}}} \leq\left\|l_{2}(t) h_{1}\right\|_{L^{p^{\prime}}}+\left\|D J^{-1} l_{1}(t) h_{2}\right\|_{L^{p^{\prime}}} \\
& \leq\left\|l_{2}(t) h_{1}\right\|_{L^{p^{\prime}}}+\left\|l_{1}(t) h_{2}\right\|_{L^{p^{\prime}}} \\
& \leq C|t|^{-\frac{n}{2}\left(\frac{2}{p}-1\right)}\left(\left\|h_{1}\right\|_{L^{p}}+\left\|h_{2}\right\|_{L^{p}}\right) .
\end{aligned}
$$

Now, the estimates (3.3) and (3.4) and a real interpolation argument yield

$$
\begin{aligned}
\left\|\pi_{1} B(t) \vec{h}\right\|_{\left(p^{\prime}, d\right)} & \leq C|t|^{-\frac{n}{2}\left(\frac{2}{p}-1\right)} \max \left\{\left\|h_{1}\right\|_{(p, d)},\left\|h_{2}\right\|_{(p, d)}\right\}, \\
\left\|\pi_{2} B(t) \vec{h}\right\|_{D^{-1} J\left(L^{\left(p^{\prime}, d\right)}\right)} & \leq C|t|^{-\frac{n}{2}\left(\frac{2}{p}-1\right)} \max \left\{\left\|h_{1}\right\|_{(p, d)},\left\|h_{2}\right\|_{(p, d)}\right\}
\end{aligned}
$$

for all $t \neq 0$ and $\vec{h} \in L^{(p, d)} \times L^{(p, d)}$. The inequalities (3.5) and (3.6) together are equivalent to (3.1).

Remark 3.1. For $1<p \leq 2$, notice that estimates (3.3) and (3.4) together imply

$$
\|B(t) \vec{h}\|_{X_{\left(p^{\prime}, p^{\prime}\right)}} \leq C|t|^{-\frac{n}{2}\left(\frac{2}{p}-1\right)}\|\vec{h}\|_{(p, p)} .
$$

We will also need the following numerical result.

Lemma 3.2. Let $0<T<\infty, 1<\rho<\gamma(n), \beta=n(\rho-1) / 2(\rho+1), \alpha=$ $1 /(\rho-1)-n / 2(\rho+1)$ and $\alpha \leq \sigma<\infty$. Denote

$$
I_{\sigma}(T)=\sup _{t \geq T} t^{\sigma} \int_{t}^{\infty}\left|t-t^{\prime}\right|^{-\beta}\left(t^{\prime}\right)^{-\sigma \rho} d t^{\prime}
$$

and

$$
H_{\sigma}(T)=\sup _{t \geq T} t^{\sigma-\beta} .
$$

Then $I_{\sigma}(T)<\infty$, and we have that

$$
I_{\sigma}(T) \rightarrow 0 \text { as } T \rightarrow \infty, \quad \text { for } \alpha<\sigma<\infty,
$$

and

$$
\sup _{T \geq 1} H_{\sigma}(T) \leq 1, \quad \text { for } \sigma<\beta
$$


Proof. First, it is clear that $1<\rho<\gamma(n)$ implies $\beta<1$ and $\alpha>0$. The change of variable $t^{\prime}=s t$ yields

$$
t^{\sigma} \int_{t}^{\infty}\left|t-t^{\prime}\right|^{-\beta}\left(t^{\prime}\right)^{-\sigma \rho} d t^{\prime}=t^{\sigma+1-\sigma \rho-\beta} \int_{1}^{\infty}(s-1)^{-\beta}(s)^{-\sigma \rho} d s .
$$

Note that

$$
\sigma+1-\sigma \rho-\beta \leq \alpha+1-\alpha \rho-\beta=0
$$

with strict inequality when $\alpha<\sigma$. On the other hand,

$$
\int_{1}^{\infty}(s-1)^{-\beta}(s)^{-\sigma \rho} d s \leq \int_{1}^{2}(s-1)^{-\beta} d s+\left(\int_{2}^{\infty} s^{-\beta-\sigma \rho} d s\right) \sup _{s>2}\left(\frac{s}{s-1}\right)^{\beta}<\infty,
$$

since $\beta<1$ and $\beta+\sigma \rho \geq \beta+\alpha \rho=1+\alpha>1$. Relations (3.10) (3.12) immediately yield (3.8).

On the other hand, inequality (3.9) follows easily from the relation $\sigma<\beta$.

\section{Proofs of theorems}

In the spirit of inverse scattering theory we must look for a fixed point for the operator

$$
\Phi: \vec{w}(t) \longrightarrow-\int_{t}^{\infty} B\left(t-t^{\prime}\right) \vec{f}\left(\vec{w}\left(t^{\prime}\right)+B\left(t^{\prime}\right) \vec{h}\right) d t^{\prime} .
$$

For the sake of completeness, in the next proposition we prove that this fixed point provides a function $\vec{u}$ satisfying the integral equation (1.5) (see also [8]).

Proposition 4.1. Let $\vec{w}$ be a fixed point of the operator $\Phi$, and define

$$
\vec{u}(t) \equiv B(t) \vec{h}+\vec{w}(t) .
$$

Then $\vec{u}$ is a solution of (1.5) in the time interval $\left[T_{0}, \infty\right)$.

Proof. Assume that $\vec{w}(t)=-\int_{t}^{\infty} B\left(t-t^{\prime}\right) \vec{f}\left(\vec{w}\left(t^{\prime}\right)+B\left(t^{\prime}\right) \vec{h}\right) d t^{\prime}$. It follows from (4.1) and the group property satisfied by $B(t)$ that

$$
\begin{aligned}
B\left(T_{0}-t\right) \vec{w} & =-\int_{t}^{\infty} B\left(T_{0}-t^{\prime}\right) \vec{f}\left(\vec{w}\left(t^{\prime}\right)+B\left(t^{\prime}\right) \vec{h}\right) d t^{\prime} \\
& =\vec{w}\left(T_{0}\right)+\int_{T_{0}}^{t} B\left(T_{0}-t^{\prime}\right) \vec{f}\left(\vec{w}\left(t^{\prime}\right)+B\left(t^{\prime}\right) \vec{h}\right) d t^{\prime} \\
& =\vec{w}\left(T_{0}\right)+\int_{T_{0}}^{t} B\left(T_{0}-t^{\prime}\right) \vec{f}\left(\vec{u}\left(t^{\prime}\right)\right) d t^{\prime} .
\end{aligned}
$$

An application of the operator $B\left(t-T_{0}\right)$ in the equality (4.2) yields

$$
\vec{w}(t)=B\left(t-T_{0}\right) \vec{w}\left(T_{0}\right)+\int_{T_{0}}^{t} B\left(t-t^{\prime}\right) \vec{f}\left(\vec{u}\left(t^{\prime}\right)\right) d t^{\prime} .
$$

Next, we add the term $B(t) \vec{h}$ on both sides of (4.3) and afterwards use the group property to obtain

$$
\begin{aligned}
B(t) \vec{h}+\vec{w}(t) & =B(t) \vec{h}+B\left(t-T_{0}\right) \vec{w}\left(T_{0}\right)+\int_{T_{0}}^{t} B\left(t-t^{\prime}\right) \vec{f}\left(\vec{u}\left(t^{\prime}\right)\right) d t^{\prime} \\
& =B\left(t-T_{0}\right)\left[B\left(T_{0}\right) \vec{h}+\vec{w}\left(T_{0}\right)\right]+\int_{T_{0}}^{t} B\left(t-t^{\prime}\right) \vec{f}\left(\vec{u}\left(t^{\prime}\right)\right) d t^{\prime} .
\end{aligned}
$$


Employing (4.1) again, the equality (4.4) becomes

$$
\vec{u}(t)=B\left(t-T_{0}\right) \vec{u}\left(T_{0}\right)+\int_{T_{0}}^{t} B\left(t-t^{\prime}\right) \vec{f}\left(\vec{u}\left(t^{\prime}\right)\right) d t^{\prime},
$$

which is equivalent to the integral equation (1.5).

Proof of Theorem 2.1. Let $a>0$ be a fixed real number and define the following closed subset of a complete metric space:

$$
X_{T}(0, a)=\left\{\begin{array}{l}
\vec{w} \in \mathcal{E}_{\sigma}^{T}: \\
\|\vec{w}\|_{\mathcal{E}_{\sigma}^{T}} \equiv \sup _{t \geq T} t^{\sigma}\|\vec{w}(t)\|_{X_{(\rho+1, \infty)}} \leq a
\end{array}\right\},
$$

where $T>0$ will be chosen later. We wish to find $T>0$ such that $\Phi$ is a contraction mapping from $X_{T}(0, a)$ to itself in the $\|\cdot\|_{\mathcal{E}_{\sigma}^{T}}$-metric. Writing $\vec{v}(t)=\left(v_{1}(t), v_{2}(t)\right) \equiv$ $\vec{w}(t)+B(t) \vec{h}$, it follows from Lemma 3.1 and the Hölder inequality that

$$
\begin{aligned}
\|\Phi(\vec{w})(t)\|_{X_{(\rho+1, \infty)}} & \leq C \int_{t}^{\infty}\left|t-t^{\prime}\right|^{-\beta}\left\|v_{1}\left(t^{\prime}\right)\right\|_{(\rho+1, \infty)}^{\rho} d t^{\prime} \\
& \leq C\|\vec{v}\|_{\mathcal{E}_{\sigma}^{T}}^{\rho} \int_{t}^{\infty}\left|t-t^{\prime}\right|^{-\beta}\left(t^{\prime}\right)^{-\sigma \rho} d t^{\prime} .
\end{aligned}
$$

Since the previous inequality holds for all $t \geq T$, we have

$$
\|\Phi(\vec{w})\|_{\mathcal{E}_{\sigma}^{T}} \leq C I_{\sigma}(T)\|\vec{v}\|_{\mathcal{E}_{\sigma}^{T}}^{\rho},
$$

where $I_{\sigma}(T)$ was given in Lemma 3.2. The definition of $\vec{v}$, Lemma 3.1 and the triangle inequality imply at once that

$$
\|\Phi(\vec{w})\|_{\mathcal{E}_{\sigma}^{T}} \leq C_{1} I_{\sigma}(T)\left(\|\vec{w}\|_{\mathcal{E}_{\sigma}^{T}}+H_{\sigma}(T)\|\vec{h}\|_{\left(\frac{\rho+1}{\rho}, \infty\right)}\right)^{\rho},
$$

where $H_{\sigma}(T)$ was given in Lemma 3.2. Next, we recall $\vec{v}(t) \equiv \vec{w}(t)+B(t) \vec{h}$, denote $\vec{r}(t)=\left(r_{1}(t), r_{2}(t)\right) \equiv \vec{z}(t)+B(t) \vec{h}$ and employ Lemma 3.1 to obtain

$$
\|\Phi(\vec{w})(t)-\Phi(\vec{z})(t)\|_{X_{(\rho+1, \infty)}} \leq C \int_{t}^{\infty}\left|t-t^{\prime}\right|^{-\beta}\left\|f\left(v_{1}\left(t^{\prime}\right)\right)-f\left(r_{1}\left(t^{\prime}\right)\right)\right\|_{X_{\left(\frac{\rho+1}{\rho}, \infty\right)}} d t^{\prime} .
$$

Recalling $\left(\left|b_{1}\right|^{\rho-1}+\left|b_{2}\right|^{\rho-1}\right) \leq 2\left(\left|b_{1}\right|+\left|b_{2}\right|\right)^{\rho-1}$, invoking (1.2), and again using the Hölder inequality, we estimate

$$
\begin{aligned}
& \|\Phi(\vec{w})-\Phi(\vec{z})\|_{\mathcal{E}_{\sigma}^{T}} \\
& \quad \leq C I_{\sigma}(T)\|\vec{w}-\vec{z}\|_{\mathcal{E}_{\sigma}^{T}}\left(\|\vec{v}\|_{\mathcal{E}_{\sigma}^{T}}^{\rho-1}+\|\vec{r}\|_{\mathcal{E}_{\sigma}^{T}}^{\rho-1}\right) \\
& \quad \leq C I_{\sigma}(T)\|\vec{w}-\vec{z}\|_{\mathcal{E}_{\sigma}^{T}} 2\left(\|\vec{v}\|_{\mathcal{E}_{\sigma}^{T}}+\|\vec{r}\|_{\mathcal{E}_{\sigma}^{T}}\right)^{\rho-1} \\
& \quad \leq C_{2} I_{\sigma}(T)\|\vec{w}-\vec{z}\|_{\mathcal{E}_{\sigma}^{T}}\left(\|\vec{w}\|_{\mathcal{E}_{\sigma}^{T}}+\|\vec{z}\|_{\mathcal{E}_{\sigma}^{T}}+2 H_{\sigma}(T)\|\vec{h}\|_{\left(\frac{\rho+1}{\rho}, \infty\right)}\right)^{\rho-1} .
\end{aligned}
$$

$$
\max \left\{C_{1} I_{\sigma}\left(T_{0}\right)\left(a+\|\vec{h}\|_{\left(\frac{\rho+1}{\rho}, \infty\right)}\right)^{\rho}, C_{2} I_{\sigma}\left(T_{0}\right) 2^{\rho-1}\left(a+\|\vec{h}\|_{\left(\frac{\rho+1}{\rho}, \infty\right)}\right)\right\} \leq \min \left\{a, \frac{1}{2}\right\} .
$$

In view of (4.5), (4.7), and (4.8), $\Phi$ is a contraction in $X_{T_{0}}(0, a)$, and therefore it has a unique fixed point, which is denoted by $\vec{w}$. 
Next we show the limit (2.7). By Proposition 4.1, $\vec{u}(t) \equiv B(t) \vec{h}+\vec{w}(t)$ satisfies the integral equation (1.5) in the time interval $\left[T_{0}, \infty\right)$. Since $\|\phi\|_{\mathcal{E}_{\sigma}^{T}} \leq\|\phi\|_{\mathcal{E}_{\sigma}^{T_{0}}}$ for all $t \geq T_{0}$, inequalities (4.5) and (4.8) yield

$$
\begin{aligned}
\|\vec{w}\|_{\mathcal{E}_{\sigma}^{T}}=\|\Phi(\vec{w})\|_{\mathcal{E}_{\sigma}^{T}} & \leq C_{1} I_{\sigma}(T)\left(\|\vec{w}\|_{\mathcal{E}_{\sigma}^{T}}+H_{\sigma}(T)\|\vec{h}\|_{\left(\frac{\rho+1}{\rho}, \infty\right)}\right)^{\rho} \\
& \leq \frac{I_{\sigma}(T)}{I_{\sigma}\left(T_{0}\right)} C_{1} I_{\sigma}\left(T_{0}\right)\left(a+\|\vec{h}\|_{\left(\frac{\rho+1}{\rho}, \infty\right)}\right)^{\rho} \\
& \leq \frac{I_{\sigma}(T)}{2 I_{\sigma}\left(T_{0}\right)} .
\end{aligned}
$$

Recalling $0<\alpha<\sigma$, we conclude that

$$
\begin{aligned}
T^{\sigma}\|\vec{u}(T)-B(T) \vec{h}\|_{X_{(\rho+1, \infty)}} & =T^{\sigma}\|\vec{w}(T)\|_{X_{(\rho+1, \infty)}} \\
& \leq \sup _{t \geq T} t^{\sigma}\|\vec{w}(t)\|_{X_{(\rho+1, \infty)}} \\
& =\|\vec{w}\|_{\mathcal{E}_{\sigma}^{T}} \leq \frac{I_{\sigma}(T)}{2 I_{\sigma}\left(T_{0}\right)} \longrightarrow 0 \text { as } T \rightarrow \infty
\end{aligned}
$$

Finally, we deal with the uniqueness assertion. Let $\vec{u}, \vec{v} \in \mathcal{E}_{\sigma}^{T_{0}}$ be two solutions satisfying (1.5) and corresponding to the same profile $\vec{h}$. Write $\vec{w}_{1}(t)=\vec{u}(t)-B(t) \vec{h}$ and $\vec{w}_{2}(t)=\vec{v}(t)-B(t) \vec{h}$. It follows from (4.6) that

$$
\begin{aligned}
\left\|\vec{w}_{1}-\vec{w}_{2}\right\|_{\mathcal{E}_{\sigma}^{T}} & =\left\|\Phi\left(\vec{w}_{1}\right)-\Phi\left(\vec{w}_{2}\right)\right\|_{\mathcal{E}_{\sigma}^{T}} \\
& \leq C I_{\sigma}(T)\left\|\vec{w}_{1}-\vec{w}_{2}\right\|_{\mathcal{E}_{\sigma}^{T}}\left(\|\vec{u}\|_{\mathcal{E}_{\sigma}^{T}}+\|\vec{v}\|_{\mathcal{E}_{\sigma}^{T}}\right)^{\rho-1},
\end{aligned}
$$

for $T \geq T_{0}$. Let $b=\max \left\{\|\vec{u}\|_{\mathcal{E}_{\sigma}^{T_{0}}},\|\vec{v}\|_{\mathcal{E}_{\sigma}^{T_{0}}}\right\}$ and take $T \geq T_{0}$ such that

$$
C I_{\sigma}(T) 2^{\rho-1} b^{\rho-1} \leq 1 / 2
$$

In view of (4.13), (4.14) we obtain

$$
\left\|\vec{w}_{1}-\vec{w}_{2}\right\|_{\mathcal{E}_{\sigma}^{T}} \leq \frac{1}{2}\left\|\vec{w}_{1}-\vec{w}_{2}\right\|_{\mathcal{E}_{\sigma}^{T}},
$$

which implies that $\vec{w}_{1}(t)=\vec{w}_{2}(t)$ in $[T, \infty)$, and so $\vec{u}(t)=\vec{v}(t)$ in $[T, \infty)$.

Proof of Theorem 2.2. The proof is parallel to that of Theorem 2.1. Indeed, it is sufficient to replace the terms $\|\vec{h}\|_{\left(\frac{\rho+1}{\rho}, \infty\right)}$ and $H_{\sigma}(T)\|\vec{h}\|_{\left(\frac{\rho+1}{\rho}, \infty\right)}$ by $\|\vec{h}\|_{\mathcal{E}_{\sigma}^{T}}$ throughout the above proof and note that (4.11), (4.12) imply that

$$
T^{\sigma}\|\vec{u}(T)-B(T) \vec{h}\|_{X_{(\rho+1, \infty)}} \longrightarrow 0 \text { as } T \rightarrow \infty .
$$

Proof of Theorem 2.3. From a fixed argument point, we know that $\vec{w}(t)$ is the limit in $X_{T_{0}}(0, a)$ of the Picard sequence $\left\{\vec{w}_{m}(t)\right\}_{m \geq 1}$

$$
\vec{w}_{1}(t) \equiv 0
$$

and

$$
\vec{w}_{m+1}(t)=-\int_{t}^{\infty} B\left(t-t^{\prime}\right) \vec{f}\left(\vec{w}_{m}\left(t^{\prime}\right)+B\left(t^{\prime}\right) \vec{h}\right) d t^{\prime} .
$$

First, denote $\|\cdot\|_{\mathcal{F}_{\sigma}^{T}}=\sup _{t>T} t^{\sigma}\|\cdot\|_{X_{(\rho+1, d)}}$ and notice that

$$
\|B(t) \vec{h}\|_{\mathcal{F}_{\sigma}^{T}}=\sup _{t>T} t^{\sigma}\|B(t) \vec{h}\|_{X_{(\rho+1, d)}}<\infty \text {. }
$$


Employing (3.1), the Hölder inequality with $d \neq \infty$, and proceeding as in the proof of (4.9), we obtain

$$
\begin{aligned}
\left\|\vec{w}_{m+1}\right\|_{\mathcal{F}_{\sigma}^{T}} & \leq C I_{\sigma}(T)\left(\left\|\vec{w}_{m}\right\|_{\mathcal{F}_{\sigma}^{T}}+\sup _{t>T} t^{\sigma}\|B(t) \vec{h}\|_{X_{(\rho+1, d)}}\right)^{\rho} \\
& \leq C I_{\sigma}(T)\|B(t) \vec{h}\|_{\mathcal{F}_{\sigma}^{T}}^{\rho}+C I_{\sigma}(T)\left\|\vec{w}_{m}\right\|_{\mathcal{F}_{\sigma}^{T}}^{\rho} .
\end{aligned}
$$

In view of

$$
I_{\sigma}(T) \rightarrow 0 \text { as } T \rightarrow \infty
$$

an induction argument (for $m \geq 1$ ) together with (4.17), (4.18) yields

$$
\lim _{t \rightarrow \infty} t^{\sigma}\left\|\vec{w}_{m}(\cdot, t)\right\|_{X_{(\rho+1, d)}}=0 \text { for all } m \geq 1 .
$$

Moreover, the numerical recurrence inequality (4.18) can be solved provided that $C I_{\sigma}(T)\|B(t) \vec{h}\|_{\mathcal{F}_{\sigma}^{T}}$ is small enough. Therefore, it follows from (4.19) that there is $C>0$ such that

$$
\left\|\vec{w}_{m}\right\|_{\mathcal{F}_{\sigma}^{T_{1}}}=\sup _{t>T_{1}} t^{\sigma}\left\|\vec{w}_{m}\right\|_{X_{(\rho+1, d)}} \leq C, \text { for some } T_{1} \geq T_{0}>0 \text { and all } m \geq 1 .
$$

To conclude the proof, it is sufficient to show that the sequence (4.15), (4.16) is contractive in the norm $\|\cdot\|_{\mathcal{F}_{\sigma}^{T}}$ for some $T \geq T_{1}>0$. For that matter, by using (4.20), we estimate

$$
\begin{aligned}
\left\|\vec{w}_{m+1}-\vec{w}_{m}\right\|_{\mathcal{F}_{\sigma}^{T}} & \leq C I_{\sigma}(T)\left\|\vec{w}_{m}-\vec{w}_{m-1}\right\|_{\mathcal{F}_{\sigma}^{T}} \\
& \times\left(\left\|\vec{w}_{m}\right\|_{\mathcal{F}_{\sigma}^{T_{1}}}+\left\|\vec{w}_{m-1}\right\|_{\mathcal{F}_{\sigma}^{T_{1}}}+\sup _{t>T_{1}} t^{\sigma}\|B(t) \vec{h}\|_{X_{(\rho+1, d)}}\right)^{\rho-1} \\
& \leq \frac{1}{2}\left\|\vec{w}_{m}-\vec{w}_{m-1}\right\|_{\mathcal{F}_{\sigma}^{T}},
\end{aligned}
$$

provided that $T$ is large enough, which yields the desired claim.

\section{REFERENCES}

1. J. Bergh and J. Lofstrom, Interpolation Spaces, Springer-Verlag, Berlin-New York, 1976. MR0482275 (58:2349)

2. J.L. Bona and R.L. Sachs, Global existence of smooth solutions and stability theory of solitary waves for a generalized Boussinesq, Comm. Math. Phys. 118 (1988), 15-29. MR954673 (89k:35198)

3. J. Boussinesq, Théorie des ondes et de remous qui se propagent le long d'un canal rectangulaire horizontal, en communiquant au liquide dans ce canal des vitesses sensiblement de la surface au fond, J. Math. Pures Appl. 17 (1872), 55-108.

4. Y. Cho and T. Ozawa, On small amplitude solutions to the generalized Boussinesq equations, Discrete Contin. Dyn. Syst. A 17 (2007), 691-711. MR2276469 (2008c:35268)

5. Y. Cho and T. Ozawa, Global existence on nonlinear Schrödinger-IMBq equations, J. Math. Kyoto Univ. 46 (3) (2006), 535-552. MR.2311358 (2008k:35437)

6. R. Côte, Large data wave operator for the generalized Korteweg-de Vries equations. Differential Integral Equations 19 (2) (2006), 163-188. MR2194502(2006i:35309)

7. F. Falk, E. Laedke, and K. Spatschek, Stability of solitary-wave pulses in shape-memory alloys, Phys. Rev. B 36 (6) (1987), 3031-3041.

8. L.G. Farah, Large data asymptotic behaviour for the generalized Boussinesq equation, Nonlinearity 21 (2008), 191-209. MR2384545 (2008m:35303)

9. L.G. Farah, Local solutions in Sobolev spaces with negative indices for the "good" Boussinesq equation, Comm. Partial Diff. Eq. 34 (2009), 52-73. MR2512853(2010k:35404) 
10. L.G. Farah, Local solutions in Sobolev spaces and unconditional well-posedness for the generalized Boussinesq equation, Commun. Pure Appl. Anal. (8) (2009), 1521-1539. MR 2505284 (2010f:35259)

11. J. Ginibre, T. Ozawa and G. Velo, On the existence of the wave operator for a class of nonlinear Schrödinger equations, Ann. IHP-Phys. Théor. 60 (1994), 211-39. MR.1270296 (95c:35232)

12. J. Ginibre and G. Velo, Long range scattering for some Schrödinger related nonlinear systems, preprint, arXiv:math/0412430v1, 2004.

13. F. Linares, Global existence of small solutions for a generalized Boussinesq equation, J. Differential Equations 106 (2) (1993), 257-293. MR.1251854(94k:35262)

14. F. Linares and M. Scialom, Asymptotic behavior of solutions of a generalized Boussinesq type equation, Nonlinear Anal. 25 (11) (1995), 1147-1158. MR1350736 (96h:35189)

15. Y. Liu, Decay and scattering of small solutions of a generalized Boussinesq equation, J. Funct. Anal. 147 (1) (1997), 51-68. MR1453176 (98e:35144)

16. D. H. Peregrine, Equations for water waves and the approximations behind them, Waves on Beaches and Resulting Sediment Transport, Academic Press, New York, 1972, 95-122.

17. A. Shimomura, Scattering theory for the Schrödinger-improved Boussinesq system in two space dimensions. Asymptot. Anal. 51 (2) (2007), 167-187. MR2311159 (2008g:35150)

18. M. Tsutsumi and T. Matahashi, On the Cauchy problem for the Boussinesq type equation, Math. Japon. 36 (2) (1991), 371-379. MR1095753 (91k:35235)

19. S. Xia and J. Yuan, Existence and scattering of small solutions to a Boussinesq type equation of sixth order, Nonlinear Anal. 73 (4) (2010), 1015-1027. MR2653768 (2011e:35283)

Departamento de Matemática, Universidade Federal de Minas Gerais, Caixa Postal 702, CEP 30161-970, Belo Horizonte-MG, Brazil

E-mail address: lgfarah@gmail.com

Departamento de Matemática, Universidade Estadual de Campinas, CEP 13083-859, CAMPINAS-SP, BRAZIL

E-mail address: lcff@ime.unicamp.br 